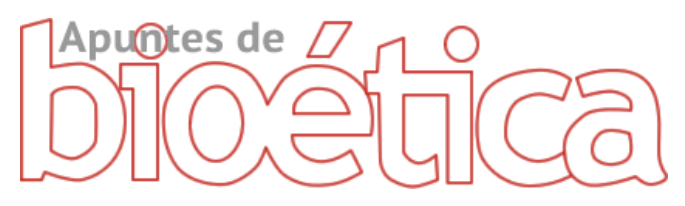

https://doi.org/10.35383/apuntes.v4i2.669 e-ISSN:2663-4910

Universidad Católica Santo Toribio de Mogrovejo

\title{
Situación actual de la salud mental de las mujeres en el Perú
}

\author{
Current situation of mental health of women in Peru
}

\author{
Susan Guadalupe Sanchez Piscoya ${ }^{*}{ }^{1}$ \\ guadalupe_10_15@hotmail.com \\ https://orcid.org/0000-0001-7294-4885
}

\author{
*Autor corresponsal \\ ${ }^{1}$ Estudio Jurídico Suel. Chiclayo, Perú \\ a Magister en Persona, Familia y Matrimonio
}

\author{
Fechas importantes \\ Recibido: 03/10/2021 \\ Aceptado: $21 / 12 / 2021$ \\ Publicado online: $23 / 12 / 2021$
}

\section{Resumen}

En el presente trabajo se parte de la importancia de la salud mental en las mujeres peruanas, en especial, a las víctimas de violencia (física, psicológica, sexual) y en situación de vulnerabilidad (mujeres en situación de abandono en instituciones mentales). Para conocer la situación actual de la salud mental de las mujeres en el Perú, hemos estudiado la normativa nacional, el modelo implementado, el presupuesto designado para su cumplimiento, y los resultados de estudios epidemiológicos, estadísticos y supervisiones. Estamos convencidos de la importancia de la atención de la salud mental de la mujer y de su entorno, así como de la importancia de acciones de concientización, promoción, prevención y tratamiento de la salud mental del Estado junto con los demás sectores, con participación activa de la comunidad, que permitan la reinserción social de la persona, para así desarrollarnos en una sociedad que cuida su salud mental y la de su familia.

Palabras clave: Salud Mental; Salud Mental en Mujeres; Abandono en Instituciones Psiquiátricas; Violencia en la Mujer. 


\begin{abstract}
This work starts from the importance of mental health in Peruvian women, especially victims of violence (physical, psychological, sexual) and in vulnerable situations (women abandoned in mental institutions).In order to know the current situation of the mental health of women in Peru, we have studied the national regulations, the implemented model, the budget designated for its compliance, and the results of epidemiological, statistical and supervisory studies. We are convinced of the importance of mental health care for women and their environment, as well as the importance of awareness actions, promotion, prevention and treatment of mental health of the State together with other sectors, with active participation of the community, that allow the social reintegration of the person, in order to develop ourselves in a society that takes care of their mental health and that of their family.
\end{abstract}

Keywords: Mental Health; Mental Health in Women; Abandonment in Psychiatric Institutions; Violence against Women.

\title{
Introducción
}

La salud mental es la construcción y desarrollo continúo asociado a la vivencia social, laboral, familiar y comunitaria, se asocia al nivel de satisfacción material y espiritual, permitiendo el desarrollo físico, intelectual, emocional, óptimo de la persona y en su proyecto de vida (Ticona, 2014; Custo, 2008).

En sentido negativo, la ausencia de salud mental vendría a ser la ausencia de salud emocional, física e intelectual, que no permite el normal desarrollo de la persona, afectándola en su ámbito social, laboral, familiar y comunitario, siendo importante, que ante ello, el Estado realice acciones de prevención, brinde tratamiento de calidad y oportuno.

En nuestro país las mujeres son las principalmente afectadas por enfermedades mentales, las cuales son causadas mayormente por violencia (física, psicológica y sexual), ejercida muchas veces por su pareja en la etapa adolescente, afectando su desarrollo cognitivo y físico, y el de su entorno, en especial, a sus hijos, los cuales también son afectados en su salud mental.

Otra grave afectación a la salud mental de la mujer, es el abandono que sufre en las instituciones psiquiátricas, en las cuales son internadas para su atención, sin embargo, las hospitalizan por periodos prolongados sin tener en cuenta el tratamiento adecuado para la enfermedad que sufren, perdiendo la habilidad de socializar. Pese que el Estado, ha venido 
implementando el modelo de atención comunitario y centros de atención comunitario para su traslado, atención inmediata, tratamiento y reinserción a la sociedad.

El presente artículo tiene como objetivo general conocer la situación actual de la salud mental de las mujeres en el Perú, para ello se ha recopilado información de las instituciones psiquiátricas, estudios epidemiológicos, supervisiones realizadas por Defensoría del Pueblo y encuestas nacionales. Los objetivos específicos son conocer: i) Normativa en salud mental, ii) Afectación de la violencia en la salud mental de la mujer iii) Estado de las mujeres en las instituciones psiquiátricas.

\section{Situación actual de la salud mental de las mujeres en el Perú}

La salud mental es un derecho reconocido en el artículo $7^{\circ}$ de nuestra Carta Magna, señala que la persona que sufra una deficiencia física o mental, se le debe respetar su dignidad, proteger legalmente, atender, readaptar y brindar seguridad; el Tribunal Constitucional (en adelante TC) ha desarrollado el contenido de este derecho y las acciones que debe tomar el Estado, como se detalla a continuación.

El TC en el Expediente $\mathrm{N}^{\circ}$ 02480-2008-PA/TC, el cual trata sobre un paciente con esquizofrenia paranoide, al cual se recomienda darle de alta, pese a que la madre de este, no cuenta con recursos ni condiciones de salud, ante ello el TC, considera pertinente para resolver la pretensión, abordar el contenido del derecho a la salud mental, y la forma en que el Estado debe atender estos requerimientos.

Para el TC, La salud mental es un derecho fundamental, su finalidad es la protección de la salud, integridad personal y condiciones dignas, comprendiendo este derecho: el acceder a tratamientos adecuados e idóneos, atención medica integral (diagnostico, medicamento, tratamiento, rehabilitación e intervenciones quirúrgicas, etc.); para ello, el Estado debe crear condiciones necesarias como son: establecimientos suficientes, medicamentos, acceso oportuno a los servicios, entre otros, para su atención (Expediente No 02480-2008-PA/TC). Se debe asignar el presupuesto suficiente, para que se cumpla con las condiciones necesarias para atender este derecho. 
Conociendo la protección constitucional que se le da a la salud mental y las condiciones necesarias de este derecho, que debe cumplir el Estado, mencionadas en el párrafo anterior, a continuación, tocaremos los subtemas respecto a la salud mental de las mujeres, como son: normativa de la salud mental en mujeres, afectación de la violencia en la salud mental de la mujer y estado de las mujeres en las instituciones psiquiátricas, lo cual, nos permitirá conocer si es efectiva su protección normativa, el estado actual de su salud mental y la realidad en las instituciones psiquiátricas.

\section{Normativa en Salud mental}

Como se mencionó anteriormente en el artículo $7^{\circ}$ de nuestra Carta Magna, se señala de manera específica la protección de la salud mental por el Estado y que este debe conducir la política en salud mental en su artículo $9^{\circ}$, siendo el ente encargado de supervisar ello el Ministerio de Salud, el cual la regula, conduce y promueve.

Para la atención de la salud mental el Estado debe crear condiciones necesarias como son: establecimientos suficientes, medicamentos, acceso oportuno a los servicios, entre otros. (Expediente $\mathrm{N}^{\circ}$ 02480-2008-PA/TC).

A nivel normativo en 1997 se promulgó la Ley No 26842 - Ley General de Salud, la cual señala expresamente en concordancia con lo señalado en la constitución, que toda persona tiene el derecho a gozar del más alto nivel de salud mental, se debe garantizar programas y servicios en número suficiente a nivel nacional, además de la promoción, prevención, recuperación y rehabilitación, el acceso a este servicio sea adecuado y de calidad.

Pese a lo señalado en la Ley General de Salud respecto de la protección de la salud mental entre ellas el de la mujer, no se contaba desde 1997 con una norma específica de salud mental (Defensoría del Pueblo, 2018), solo con una norma general, por lo que, la salud mental no contaba con propio presupuesto para su atención, sino estaba supeditada al presupuesto nacional de salud, el cual solo destinaba el $1 \%$ para ello, pese a la alta prevalencia enfermedades mentales en mujeres, además no se contaba con las condiciones necesarias para prestar este servicio (falta de medicamentos, pocos establecimientos, brecha para acceder a los servicios). 
Posteriormente en el año 2004, se adopta el modelo de atención comunitaria, que reconoce la autonomía y la dignidad de la persona con problemas mentales, su reinserción a la sociedad, prioriza la atención ambulatoria e inmediata, dejando de lado la atención de los pacientes mediante largos periodos de internamiento, a través de la atención en centros comunitarios, de manera descentralizada, y de acuerdo a la necesidad de cada territorio.

Sin embargo, pese a la adopción del modelo comunitario en 2004 es recién que en 2012 que se comenzó a implementar los mencionados centros (Defensoría del Pueblo, 2018), concentrándose en su mayoría en la capital y zona norte del país, no cumpliéndose con su implementación a nivel nacional; y con lo señalado por el TC en el Expediente $\mathrm{N}^{\circ}$ 024802008-PA/TC respecto de las condiciones necesarias para acceder a la atención de la salud mental, al no contar con establecimientos suficientes (centros comunitarios) a nivel nacional.

Recientemente en el año 2019, se promulgó la Ley No 30947 - Ley de salud mental, que señala de manera específica la protección y atención de la salud mental de las mujeres; y que se debe priorizar su atención, en especial a las que son víctimas de violencia (física, psicológica, sexual) y en estado de vulnerabilidad; debiéndose poner énfasis en la prevención, y en el acceso a los servicios de salud mental de manera oportuna, además, se cuenta con el apoyo del Ministerio de la Mujer y Poblaciones Vulnerables para la búsqueda de los familiares de las mujeres que se encuentren a la vez en situación de abandono o desprotección, el cual coordina conjuntamente con los ministerios de los demás sectores.

La mencionada ley en su artículo $10^{\circ}$ señala que tanto en los seguros públicos y privados se debe atender la salud mental de los pacientes, el cual incluye tratamiento ambulatorio, internamiento, hospitalización, medicamento.

Además señala que las personas hospitalizadas innecesariamente deben ser derivadas a profesionales de trabajo social o especialidades afines, para ser reinsertadas en la sociedad y comunidad. Si se encuentran en situación de abandono una vez terminado su tratamiento el responsable del establecimiento coordina con el Ministerio de la Mujer, para la búsqueda de sus familiares, en caso de no ser encontrados, se les traslada a hogares protegidos.

Pese a tener una ley específica en salud mental, hasta la actualidad, se continúa destinando solo del presupuesto de salud el $1 \%$ a la salud mental, lo que no permite, la atención 
suficiente de la salud mental y la designación de presupuesto necesario para el Plan Nacional contra la violencia hacia la mujer y Plan Nacional de Acción por la Infancia y la Adolescencia 2012-2021, que buscan ayudar, sensibilizar y brindar tratamiento a la mujer y sus menores hijos.

La realidad nacional de nuestro país respecto a la salud metal de la mujer, su protección, prevención, cuidado y tratamiento varía a lo señalado en la norma, pese a contar con una Legislación de Salud Mental que protege expresamente a la mujer; no se cuenta con presupuesto y centros comunitarios suficientes para su atención; la salud mental de las mujeres se ha visto a afectada principalmente por violencia (física, psicológica, sexual) y abandono en las instituciones psiquiátricas por parte de sus familiares, lo que requiere inmediata atención por parte del Estado, como se detalla a continuación.

\section{Violencia contra la mujer}

Nuestro país tiene una alta prevalencia en violencia, muestra de ello, son los resultados de la Encuesta Demográfica y de Salud Familiar del año 2000, según la cual más 20\% eran amenazadas, más del 30\% habían sido sometidas a situaciones de control, más del $40 \%$ de mujeres a humillaciones, agredidas físicamente y violencia psicológica por su pareja o esposo (Instituto Nacional de Estadística e Informática, 2000), evidenciándose la necesidad de la atención de su salud mental, así como su tratamiento y prevención.

Dicha situación ha empeorado a través de los años, el Estudio Epidemiológico Metropolitano de Salud Mental 2002 señala que la prevalencia de la depresión era mayor en mujeres que en hombres, las mujeres mayores a 15 años sufrían de depresión (16\%), el suicidio afectaba la prevalencia de vida (42\%) y prevalencia anual (12\%), afectando la prevalencia de vida del varón en $5 \%$ a diferencia de la mujer en un $8 \%$. La depresión que sufrían las mujeres era en su mayoría causada por celos (50\%), machismo (36\%), control de la pareja (20\%), alcoholismo (21\%) e infidelidad (19\%)(Instituto Especializado en Salud Mental,2002), siendo las enfermedades mentales mayores en mujeres que en hombres, y se presentaban desde la adolescencia.

La prevalencia de vida de la mujer ante algún tipo de abuso era mayor a 40\%, afectada principalmente por insultos (40\%), abuso físico (20\%), humillación (15\%), abandono (10\%) 
y abuso sexual (9\%) (Bravo de Rueda Ortega, 2006), lo que evidencia, que la salud mental de la mujer era afectaba principalmente por la violencia (física, psicológica y sexual) afectando su salud a largo plazo, sufriendo depresión, terminando muchas veces en suicidios.

Los estudios epidemiológicos realizados por el Instituto Nacional de Salud Mental Honorio Delgado - Hideyo Noguchi (INSM HD-HN en adelante), nos permiten conocer el estado de la salud mental de las mujeres que sufren violencia, los resultados obtenidos indicaron que para el año 2006 las ciudades de Arequipa e Ica tenían más del 50\% y Piura, Chimbote y Trujillo más del $40 \%$ de mujeres sufría un abuso, lo cual no era muy distinto a la situación de las demás ciudades del país (INSM HD-HN, 2006; Vargas, 2017).

Para el año 2010, en la capital de nuestro país, en la zona sur más del $50 \%$ y en la zona norte más del $40 \%$ de mujeres sufría algún tipo de maltrato, de estas cifras en su gran mayoría el maltrato fue ejercido por sus parejas (Instituto Nacional de Salud Mental, 2010). Para 2012, Lima Metropolitana y Callao, se evidenció una prevalencia de vida de abuso, aumentando el maltrato hacia la mujer en un 54\% (Instituto Nacional de Salud Mental, 2013).

Para el año 2018, 4 millones de personas sufrían de alguna enfermedad mental, de las cuales el $80 \%$ no eran atendidas, en especial personas en situación de pobreza y en situación de vulnerabilidad, entre ellas, las mujeres; y se preveía que para 2021 se incrementaría en $3,2 \%$ el porcentaje de personas que sufrirían enfermedades mentales (Defensoría del Pueblo, 2018).

La no atención de las enfermedades mentales oportunamente, dan como resultado que, en un mes en la capital $(1 \%)$, sierra $(0,7 \%)$ y selva $(0,6 \%)$ se den intentos suicidas (Rondón, 2006), siendo ello, un indicador, de la desatención y necesidad de priorización y tratamiento de la salud mental de las mujeres en nuestro país, mucho más con el pronóstico de incremento hacia el $2021(3,2 \%)$.

De lo mencionado anteriormente se colige que la violencia genera a largo plazo enfermedades mentales como depresión, suicido, estrés, alteraciones de la conducta y el 
aprendizaje, las cuales son prevalentes en mujeres (Instituto Canario de Igualdad, 2012) (Kessler et al., 1994), lo que afecta en su calidad de vida, bienestar y productividad.

La violencia no solo afecta a la mujer sino también a su entorno (hijos, personas cercanas), en su desarrollo físico (retraso del crecimiento, alteraciones psicológicas, del sueño y alimentación, entre otras), emocional (depresión, ansiedad, entre otros) cognitivo y de conducta (Instituto Canario de Igualdad, 2012), afectando en gran medida cuando los hijos están en etapa de crecimiento y adolescencia (Caqui, 2020).

Por ello, es importante la identificación y detención temprana de problemas mentales en mujeres afectadas por la violencia a fin de asegurar su tratamiento y el de su familia, para así prevenir el desarrollo de otras enfermedades mentales.

\section{Abandono en instituciones psiquiátricas}

Respecto al número de mujeres en situación de abandono en instituciones mentales, no se cuenta con una cifra exacta, sin embargo, existe información de las supervisiones realizadas por Defensoría del Pueblo, que señala que en 2018, en las instituciones psiquiátricas de la capital que atienden a las personas que sufren enfermedades mentales graves como es el Hospital Larco herrera (HLR) se tenía 124 personas en situación de abandono, de las cuales más del $60 \%$ eran mujeres, y se encontraban internadas por largos periodos por decisión judicial y abandono de sus familiares, situación que no era diferente a la del INSM HD-HN y Centro de Rehabilitación Calana ( $\mathrm{CRH})$, donde se encontraban en abandono por más de dos años (Defensoría del Pueblo,2018); no contándose con la información de los demás instituciones psiquiátricas y Centro de Rehabilitación, pese al pedido de información de la Defensoría del Pueblo.

El estado en que se encontraban era deplorable, compartían la ropa, no contaban con medicamento suficiente, y el medicamento era aplicado por largo periodos, perdiendo habilidad para socializar.

De lo mencionado, se evidencia que pese a la implementación del modelo comunitario de atención en 2004 y a la creación de centros comunitarios en 2012 los cuales se concentraban en la capital y zona norte del país (Trujillo, Chiclayo, Piura), y recibían a los pacientes 
brindándoles tratamiento con un equipo multidisciplinario; pese a la existencia de los centros comunitarios, las instituciones psiquiátricas no han cumplido con la aplicación del mencionado modelo, el cual establece la atención de los pacientes de manera ambulatoria, es decir, luego de terminado el tratamiento en las instituciones psiquiátricas, derivarlos a los centros comunitarios correspondiente para que continúen su tratamiento sin necesidad de hospitalización y reinsertarlos a la sociedad (Cabrera, Irigoyen y Arévalo, S., 2021).

A la actualidad, no se cuenta con información respecto de la situación de las mujeres con enfermedades mentales en estado de abandono en las instituciones psiquiátricas (INSM HDHN, HLR, CRH), siendo necesario que el Estado realice acciones de supervisión, derivación a centros comunitarios, búsqueda de familiares, en caso de no contar con ellos derivarlos a hogares protegidos tal como establece la Ley General de Salud y la Ley de Salud Mental, además de la revisión de sentencias judiciales que obligan al internamiento de mujeres en hospitales psiquiátricos.

De lo mencionado anteriormente, se concluye que la situación de la salud mental de la mujer en el Perú se ha visto afectada por una alta prevalencia de enfermedades mentales que afectan su desarrollo físico, emocional, cognitivo y de conducta; dichas enfermedades son generadas en su mayoría por violencia (física, psicológica, sexual) ejercidas por su pareja, comenzando en la etapa de la adolescencia (15 años), además de ello las mujeres que son tratadas en instituciones psiquiátricas se encuentran en estado de abandono por sus familiares, y algunas son internadas por disposición judicial por periodos prolongados mayores a dos años, sin embargo, para la atención de la salud mental se sigue designando poco presupuesto para su atención (1\% del presupuesto de salud), pese a contar en la actualidad con una Ley de Salud Mental (Ley No 30947) que establece la atención inmediata y prioritaria a la mujer, sobre todo en casos de violencia y vulnerabilidad (abandono).

\section{Conclusiones}

La atención de la salud mental, se encuentra estipulada en la Ley General de Salud- Ley No 26842- y la reciente Ley de Salud Mental - Ley No 30947 - la cual señala de manera específica la protección, atención y tratamiento de la salud mental de la mujer, en especial las que son víctimas de violencia (física, psicológica, sexual), y las que se encuentran en estado de vulnerabilidad, como son las mujeres en situación de abandono en las 
instituciones psiquiátricas, para brindar una mejor atención en salud mental, se empezó a implementar desde el 2004 el modelo de atención comunitario que establece la priorización de la atención de los pacientes de manera ambulatoria antes que hospitalaria, a fin de brindar una atención inmediata y oportuna.

La Situación problemática detectada, son la violencia (física, psicológica, sexual) ejercida desde la adolescencia en su mayoría por sus parejas, y abandono de mujeres en las instituciones psiquiátricas por parte de sus familiares, lo que requiere inmediata atención por parte del Estado y la necesidad de concientización a la familia y a la población respecto de la importancia del cuidado de la salud mental y el de su tratamiento inmediato ante cualquier afectación o hecho de violencia.

Las enfermedades mentales afectan la calidad de vida de la mujer, en su desarrollo físico (retraso del crecimiento, alteraciones psicológicas, del sueño y alimentación, entre otras), emocionales (depresión, ansiedad, entre otros) cognitivo y de conducta (Instituto Canario de Igualdad, 2012) afectando su salud a largo plazo, y también a la de su entorno, sobre todo a sus hijos que están en etapa de crecimiento y adolescencia.

No se cuenta con cifra exacta respecto al número de mujeres en situación de abandono en instituciones mentales, solo con la información de la supervisión realizada por la Defensoría del pueblo (2018), según la cual, en las instituciones psiquiátricas de nuestro país más del $60 \%$ eran mujeres en estado de abandono internadas por largos periodos por decisión judicial y abandono de sus familiares. El estado en que se encontraban era deplorable, compartían la ropa, no contaban con medicamento suficiente, y el medicamento era aplicado por largo periodos, perdiendo habilidad para socializar.

Las instituciones psiquiátricas no han cumplido con la aplicación del modelo comunitario, al continuar con internamientos prolongados de pacientes (en especial mujeres), pese a que tal como estipula la Ley de Salud Mental - Ley № 30947 - luego de terminado el tratamiento en las instituciones psiquiátricas, se les debe derivar a los centros comunitarios correspondientes para su atención.

La asignación de presupuesto del presupuesto para atender la salud mental ( $1 \%$ del presupuesto de salud) es insuficiente para atender la demanda de pacientes que requieren 
atención, entre ellas las mujeres víctimas de violencia y situación de abandono en instituciones psiquiátricas, existiendo una brecha de acceso del $80 \%$, además de ello, por motivo, del insuficiente presupuesto no se ha podido implementar completamente el modelo de atención comunitaria debido al poco presupuesto asignado para la apertura de centros comunitarios, contando solo con los aperturados en la capital y la zona norte de país.

Es preocupante el desamparo en la atención la salud mental de las mujeres, pese al aumento de prevalencia de enfermedades mentales y la afectación a su entorno (familia, hijos) y la evidente situación de abandono en instituciones psiquiátricas, siendo necesario, que el Estado, aumente el presupuesto destinado para la salud mental, se cumpla con el modelo de atención comunitaria y lo estipulado en la Ley General de Salud- Ley No 26842- y la reciente Ley de Salud Mental - Ley No 30947, respecto a la atención de la salud mental de la mujer.

\section{Recomendaciones}

El Ministerio de la Mujer y Poblaciones Vulnerables conjuntamente con el Ministerio de Salud, realicen acciones de promoción, prevención y atención de la salud mental de la mujer, en especial a las que son víctimas de violencia (física, psicológica, sexual), y en estado de vulnerabilidad.

El Ministerio de Economía y Finanzas designe presupuesto específico para la atención de salud mental, en atención a lo dispuesto por Ley de Salud Mental (2019), y la creación de centros comunitarios a nivel nacional, de acuerdo a la necesidad de cada territorio.

La atención de salud mental a las mujeres también debe de abarcar a su entorno (hijos, personas cercanas), a fin de prevenir del desarrollo de alteraciones físicas, emocionales, cognitivas y de conducta, que afectan a los hijos están en etapa de crecimiento y adolescencia.

El Ministerio de Salud como ente rector, cumpla con su función de recabar información a nivel nacional respecto de la atención de la salud mental de las mujeres, en especial, las que 
sufren violencia, actualizarla mensualmente, para con ello, el Estado tome decisiones respecto a las acciones, planes y programas a implementar.

El Ministerio de Salud, supervise las instituciones psiquiátricas, a fin de conocer el estado de las mujeres internadas por largos periodos por decisión judicial y abandonadas por sus familiares, así como su tratamiento, medicina y ropa comunitaria que utilizan, para con ello, se replante acciones a realizar, para su derivación a centros comunitarios, donde corresponde ser tratadas y reinsertadas a la sociedad.

Ministerio de salud supervise la implementación a nivel nacional de los centros comunitarios, en las ciudades, en las que por falta de presupuesto no fueron aperturados, así como la derivación de pacientes a estos por parte de instituciones psiquiátricas en cumplimiento del modelo comunitario.

\section{Referencias}

Bravo de Rueda, O. C. (2006). Bioética, salud mental y género. Acta bioethica, 12(2), 169-175.

Cabrera, M. E., Irigoyen, A., \& Arevalo, S. (2021). Cuidados Paliativos, una disciplina que humaniza la práctica médica al final de la vida: Algunos aportes desde la psicología. Apuntes de Bioética,4(1), 122143. https://doi.org/10.35383/apuntes.v4i1.608

Caqui Pajuelo, Y. M. (2020). Violencia familiar contra la mujer: análisis desde un enfoque personalista. Apuntes De Bioética, 3(2), 62-80. https://doi.org/10.35383/apuntes.v3i2.494

Constitución Política del Perú (1993). Promulgada el 29 de diciembre de 1993. Lima, PE, 2002.

Custo, E. (2008). Salud mental y ciudadanía: una perspectiva desde el Trabajo. Buenos Aires: Espacio editorial.

Defensoría del Pueblo (2018). Supervisión de la implementación de la política pública de atención comunitaria y el camino a la desinstitucionalización. Informe Defensorial $\mathrm{N}^{\circ} 180$. Lima.

Instituto Canario de Igualdad, Servicio de Coordinación del Sistema Integral contra la Violencia de Género del Gobierno de Canarias (2012). Guía de intervención con menores víctimas de violencia de género. Las Palmas: Instituto Canario de Igualdad; 2012.

Instituto Especializado en Salud Mental (2002). Estudio Epidemiológico Metropolitano en Salud Mental 2002. Informe General. Anales de Salud Mental 2002; 18(1-2).

Instituto Nacional de Estadística e Informática (2000). Encuesta Demográfica y de Salud FamiliarENDES. Lima: Instituto Nacional de Estadística e Informática; 2000.

Instituto Nacional de Salud Mental (2010). Estudio Epidemiológico de Salud Mental en la ciudad de Abancay 2010; Informe General. Anales de Salud Mental. 2009; 27 (1):1-302. 
Instituto Nacional de Salud Mental (2013). Estudio Epidemiológico de Salud Mental en Lima Metropolitana y Callao Replicación 2012; Informe General. Anales de Salud Mental. 2013; 29(1):1-392.

Instituto Nacional de Salud Mental Honorio Delgado - Hideyo Noguchi (2007). Estudio Epidemiológico de Salud Mental en la Costa 2006; Informe General. Anales de Salud Mental. 2007; 23 (12):1-226.

Kessler R.C., Mc Gonable K.A., Zhao S., et al (1994). Lifetime and 12-month prevalence of DSM-IIIR psychiatric disorders in the United States: Results from the National Comorbidity Survey. Arch Gen Psychiatry.1994;51:8-19.

Ley N² 26842(1997). Ley General de Salud. El Peruano. Normas Legales. 09 de Julio de 1997.

Rondón, M. B. (2006). Salud mental: un problema de salud pública en el Perú. Revista peruana de medicina experimental y salud pública, 23(4), 237-238.

Sentencia del Tribunal Constitucional emitida el 11 de julio del año 2008, respecto al Expediente $\mathrm{N}^{\circ}$ 02480-2008-PA/TC, fundamento jurídico 11.

Sentencia del Tribunal Constitucional recaída en el Expediente No 2945-2003-AA/TC.

Ticona, P. (2014). El derecho a la salud mental en el Perú del siglo XXI cun derecho protegido o un derecho postergado por el Estado peruano? Tesis para optar el grado académico de Magister en Derecho Constitucional de la Pontificia Católica del Perú.

Vargas, J. H. (2017). Violencia contra la mujer infligida por su pareja y su relación con la salud mental de los hijos adolescentes. Revista médica herediana, 28(1), 48-58.

\section{Cómo citar este trabajo}

Sanchez Piscoya, S. G. (2021). Situación actual de la salud mental de las mujeres en el Perú. Apuntes De Bioética, 4(2), 159-171. https://doi.org/10.35383/apuntes.v4i2.669

\section{Financiación}

El presente artículo no cuenta con financiación específica de agencias de financiamiento en los sectores público o privado para su desarrollo y/o publicación.

\section{Conflicto de interés}

El autor del artículo declara no tener ningún conflicto de intereses en su realización.

(C) Los autores. Este artículo es publicado por la Revista Apuntes de Bioética del Instituto de Bioética, Universidad Católica Santo Toribio de Mogrovejo.

Este es un artículo de acceso abierto, distribuido bajo los términos de la Licencia Creative Commons Atribución-NoComercial-CompartirIgual 4.0 Internacional (CC BY-NC-SA 4.0), que permite el uso no comercial, distribución y reproducción en cualquier medio, siempre que la obra original sea debidamente citada. 